Brothers and sisters born at different times, and not related to twins :- 50 pairs.

\title{
SUMMARY.
}

Cases alike or with only $0.5 \mathrm{D}$. of difference . 14 per cent. Cases approximately alike . . . . 16 per cent. Cases with more than $1 \mathrm{D}$. of difference. . 70 per cent.

In the first group here there are six squinters, in the second 4, and in the third 31 squinters.

I regret that my reports are imperfect from the biometrician's point of view.

It is highly desirable that others who have the opportunity should collect and report their observations on the state of refraction, the corneal curvature, the presence or absence of strabismus, the general resemblances and even other measurements. It is probable that such data would assist the valuable work being done by Dr. Percy Stocks and others, now in course of publication in the Annals of Eugenics.

\section{A SUGGESTION AS TO THE CAUSE OF GLAUCOMA FOLLOWING THROMBOSIS OF THE RETINAL VEIN}

BY

D. J. WOOD

CAPE TOWN

I THINK it is commonly agreed that where a patient's eyes are near the border line between normal and increased tension, the occurrence of thrombosis of the retinal vein may lead to a glaucomatous attack. The reasons for this do not seem quite satisfactory, and the following observations may be of use.

I have for a long time been in the habit of examining all the cases of fundus disease seen by me in private by red-free light, which, with a plane mirror, gives one an ideally fine picture of the finest details in the retina.

A 4.5 amp. Leitz arc lamp, a water cooler, a convex lens t. render the rays only slightly divergent, and a Zeiss red-free glass filter is all the equipment needed, and costs about $£ 5$.

In old age the yellow tint of the patient's lens dims the brilliancy of the picture, and here one can substitute a Kodak Green " $B$ " filter which will illuminate a fundus even where the lens is getting opaque. This has been used by others. It does not apply to the present cases. 
In 1929 my attention was drawn to the fact that in the fundus of a young lady who had thrombosis with many haemorrhages, though one could see clearly with the ordinary electric light, the picture by red-free light was very dim. The vitreous, though clear, would not transmit the blue end of the spectrum.

Since then I have seen eight cases, and wherever the haemorrhages were extensive, the vitreous was found to be more or less opaque to red-free light.

Four patients were under 40 years of age. In two there were many haemorrhages, but glaucoma did not supervene, presumably because of youth. In two there were few haemorrhages, and neither was the vitreous stained, nor did glaucoma occur. Five cases were seen in older people. In two the haemorrhages were few, and the vitreous appeared similar to that in the other eye. Glaucoma did not occur.

In three there were many haemorrhages, with definite dimness of the fundus in red-free light. Two developed glaucoma, but died before pain demanded excision. In the third case I had to excise the eye. I was more interested in his retina than in his vitreous, and it did not occur to me to ask a bio-chemist to test the latter for haemoglobin derivatives, as might have been possible.

By red-free light one can see in some cases that not only are there haemorrhages in the retina, there are also haemorrhages on the retina which show up as dark glistening spheres.

The suggestion derived from these few cases is that some substance formed from haemoglobin is absorbed into the vitreous, and if in sufficient quantity alters its light transmitting properties, and may cause a turgescence of the vitreous gel.

Where there are a larger number of cases available for examination one hopes this suggestion may lead to further investigation.

\title{
KANDA'S CURVED SCREW ROLLER FORCEPS (A NEW MODEL OF MODIFICATION FROM KNAPP'S ROLLER FORCEPS)
}

BY

\author{
Dr. KanjI Kanda \\ TAKAO, FORMOSA, JAPAN
}

"Expression of the granules with Knapp's Roller Forceps is" says Professor Komoto, "an international method of treatment of Trachoma." Indeed, Knapp's roller forceps, with its long history of existence since first devised by Dr. Knapp, is the most excellent instrument among those of the same design, so that it 